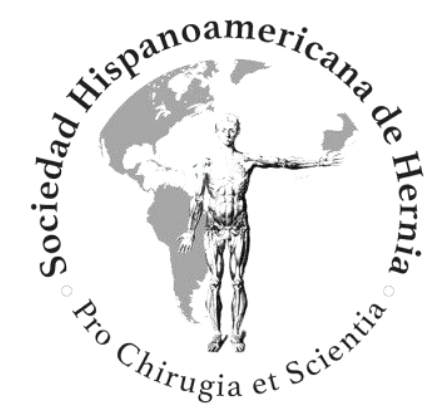

Estudio sobre la correlación entre el grosor del oblicuo externo y su fuerza mecánica

Correlating the muscle strength of the abdominal wall with the thickness of the external oblique muscle 


\section{OR 436}

\section{Estudio sobre la correlación entre el grosor del oblicuo externo y su fuerza mecánica}

Correlating the muscle strength of the abdominal wall with the thickness of the external oblique muscle

Mikel Osorio Capitán ${ }^{1}$, Igor Novo Sukia ${ }^{1}$, Maitane Larrañaga Zabaleta ${ }^{1}$, Irene Aramendia García ${ }^{1}$, María Jesús Busto Vicente', Aintzane Lizarazu Pérez², Laura Carballo Rodríguez², íñigo Augusto Ponce², Lander Gallego Otaegui ${ }^{2}$

${ }^{1}$ Servicio de Cirugía General y del Aparato Digestivo. Hospital Comarcal de Zumárraga. Zumárraga, Guipúzcoa (España). ${ }^{2}$ Servicio de Cirugía General y del Aparato Digestivo. Hospital Universitario Donostia. San Sebastián (España)

Recibido: 28-01-2021

Aceptado: 22-02-2021

Autor para correspondencia: Mikel Osorio Capitán. Servicio de Cirugía General y del Aparato Digestivo. Hospital Comarcal de Zumárraga. Barrio Argixao, s/n. 20700 Zumarraga, Guipúzcoa (España) Correo electrónico: mosorio002@gmail.com

DOI: $10.20960 /$ rhh.00436

Conflictos de interés: los autores declaran no tener conflictos de interés.

Financiaciones: los autores declaran no haber recibido apoyo financiero para la investigación, autoría y/o publicación de este artículo. 


\section{RESUMEN}

Introducción: Los nuevos conocimientos sobre la biomecánica de la pared abdominal, acompañados de procedimientos novedosos como la denervación química muscular, han impulsado un escenario que preconiza la individualización del paciente y de la técnica quirúrgica.

A mayor grosor muscular, la lógica nos sugiere mayor fuerza en la línea media, por lo que demostrar dicha premisa y poder cuantificar la fuerza muscular preoperatoriamente podría aportar una información crucial.

Con este estudio se ha tratado de correlacionar la fuerza muscular de la pared abdominal con el grosor del músculo oblicuo externo.

Material y métodos: Se procedió a un estudio descriptivo transversal con 8 individuos. El grosor del oblicuo externo se midió por medio de una imagen de TC preoperatoria. La fuerza muscular se calculó durante la operación a través de un dinamómetro.

Resultados: La correlación entre el grosor del oblicuo externo y la fuerza de la pared abdominal fue positiva, con un coeficiente de correlación de Pearson de 0.767 ( $p=0.026$; IC $95 \%$, 0.136-0.955). La correlación entre el grosor completo de la pared muscular lateral del abdomen y la suma entre el oblicuo interno y el músculo transverso arrojó resultados sin relación lineal.

Conclusión: Por medio de este estudio se ha establecido una correlación lineal entre la fuerza muscular y su imagen preoperatoria y se ha obtenido una correlación significativamente positiva entre el grosor del músculo oblicuo externo y la fuerza de la pared abdominal.

Se deduce, por tanto, que dentro de los músculos laterales del abdomen, el oblicuo externo es el que más fuerza añade a la línea media y que su tratamiento preoperatorio individualizado podría facilitar las reparaciones de la pared abdominal. Cabría esperar futuros estudios en los que se determinara a partir de qué grosor del oblicuo externo resultaría beneficioso aplicar tratamientos locales preoperatorios. 
Palabras clave: Músculo oblicuo externo (MOE), grosor de MOE, fuerza muscular del abdomen, dinamómetro.

\section{ABSTRACT}

Introduction: Recent studies about the biomechanics of the abdominal wall and the appearance of new procedures such as the chemical muscle denervation, has promoted a scenario that advocates the individualization of the patient and the surgical technique.

As the muscles thickness increase, the force exerted on de midline could be higher. Demonstrating this premise and being able to quantify muscle strength preoperatively could provide a crucial surgical information.

Through this study we have tried to correlate the muscle strength of the abdominal wall with the thickness of the external oblique muscle.

Material and methods: A descriptive cross-sectional study was carried out with eight individuals. The thickness of the external oblique was measured by means of a preoperative CT image. Muscle strength was calculated intraoperatively through a dynamometer.

Results: The correlation between the thickness of the external oblique muscle and the strength of the abdominal wall was positive, with a Pearson's correlation coefficient of $0.767(p=0.026 ; 95 \% \mathrm{Cl}$ 0.136-0.955). The correlation between the thickness of all the abdominal lateral muscles and that corresponding to the thickness of the internal oblique and the transverse muscles, yielded results with no linear relation.

Conclusion: Through this study a linear correlation between muscles strength and its preoperative image has been established, obtaining a positive significant correlation between the thickness of the external oblique muscle and the force exerted on the midline.

Therefore, as the external oblique muscle seems to have the most strength between the abdominal lateral muscles, it preoperative 
conditioning could improve the surgical results. Future studies may be expected to determine from what thickness of the external oblique muscle would be beneficial to apply preoperative local treatments.

Keywords: External oblique muscle (EOM), EOM thickness, abdominal muscles strength, dynamometer.

\section{INTRODUCCIÓN}

Cada vez que se realiza una cirugía que conlleva la apertura de la pared abdominal, ya sea por cirugía laparotómica o laparoscópica, la sección musculofascial obligada conlleva un debilitamiento de la pared abdominal propensa a futuras hernias. De hecho, a pesar de las nuevas técnicas de cierre fascial, la incidencia de la hernia se describe entorno al 15-20 $\%^{1}$ en las mejores series, con un índice de recibida después de la eventroplastia que puede ascender al $43 \%{ }^{2}$.

En este contexto, la cirugía de la pared abdominal ha presentado un desarrollo considerable en cuanto a técnicas y procedimientos quirúrgicos en los últimos años. Además, el conocimiento sobre la biomecánica de la pared abdominal y el funcionamiento de sus músculos ha facilitado el desarrollo de técnicas novedosas. Un ejemplo de ello es la aplicación de la toxina botulínica para la relajación de la fibra muscular ${ }^{3}$.

La función de los músculos de la pared abdominal, enigmática en muchos aspectos, contribuye a los movimientos respiratorios y a la contención de las vísceras abdominales ${ }^{7-9}$. Aunque la acción sinérgica entre los músculos oblicuo interno (MOI), transverso y diafragma facilita una correcta dinámica ventilatoria ${ }^{7}$, la contención visceral abdominal nace predominantemente de la función del oblicuo externo (MOE), en concordancia con la contracción del recto abdominal y del MOI contralateral ${ }^{8,9}$.

Por otro lado, la toxina botulínica funciona como agonista competitivo de la acetilcolina, lo que afecta a su unión con sus 
receptores musculares y provoca una parálisis flácida de la fibra muscular. Por medio de su acción neurotóxica se consigue una relajación y una elongación de los músculos abdominales ${ }^{3}$, lo que facilita sucesivas reparaciones.

Desde que Ibarra y cols. ${ }^{3}$ en 2009 describieran la posible aplicación de la toxina botulínica para la cirugía de la pared abdominal, su extensión se ha globalizado, con resultados prometedores ${ }^{4,5}$. Hasta ahora su aplicación ha estado limitada a grandes eventraciones con pérdida de derecho a domicilio ${ }^{4}$, pero su posible correlación dosisdependiente, asociada a la idea innovadora de modificar su dosis según el grosor muscular ${ }^{6}$, presenta un nuevo escenario favorable para modificar su indicación de manera más selectiva y extenderla a un mayor número de pacientes.

De todo lo mencionado previamente puede inferirse que la relajación química del MOE podría ofrecer una medialización de los músculos abdominales con una probable preservación de la mecánica respiratoria. Además, la dosificación dependiente del grosor muscular ${ }^{6}$ hace suponer que, a mayor grosor y cantidad de fibra muscular, la fuerza ejercida sobre la línea media será mayor. Pendiente de demostrar la relación entre el grosor y la fuerza muscular, la indicación de la aplicación de la toxina botulínica o la elección de la técnica quirúrgica podrían seleccionarse para pacientes cuya fuerza mecánica muscular abdominal fuese elevada (y puede modularse para obtener resultados más eficientes).

Por medio de este estudio se ha tratado de correlacionar la fuerza del paquete muscular lateral del abdomen con su grosor radiológico con objeto de obtener un parámetro preoperatorio objetivo de la fuerza de la pared abdominal de cada paciente.

\section{MATERIAL Y MÉTODOS}

Se trata de un estudio transversal y descriptivo de pacientes intervenidos de hernia primaria o de eventración en el Hospital Comarcal de Zumárraga (Zumárraga, Guipúzcoa, España) entre los 
meses de agosto y octubre del 2020. Las reparaciones incluidas fueron todas aquellas realizadas por medio de un abordaje abierto laparotómico. Los criterios de exclusión fueron: eventraciones lumbares, subcostales, eventraciones de la línea media próximas al xifoides (M1 según la clasificación de la European Hernia Society, EHS), eventraciones complejas con orificios sincrónicos distantes de la línea media, pacientes sin pruebas de imagen preoperatoria en los últimos 12 meses y reparaciones laparoscópicas.

Los parámetros descritos y analizados fueron: edad, comorbilidades asociadas (enfermedad pulmonar obstructiva crónica [EPOC], diabetes mellitus [DM], tabaquismo y tratamiento inmunosupresor), malnutrición proteica (analítica preoperatoria), índice de masa corporal (IMC), fuerza muscular intraoperatoria (newton) y mediciones radiológicas $(\mathrm{cm})$ de los músculos abdominales: grosor de $\mathrm{MOE}$, grosor de $\mathrm{MOI}$ + transverso, grosor de paquete muscular lateral del abdomen, defecto herniario y diástasis de músculos rectos del abdomen (siempre que esta existiera).

La $n$ final analizada fue de 8 individuos, 4 pacientes, suponiendo cada paciente 2 individuos independientes por cada hemiabdomen analizado.

Todos los datos estadísticos se manejaron por medio del programa GraphPad. Habiéndose obtenido variables cuantitativas y continuas, la correlación estadística se trató de hallar por medio del coeficiente de correlación de Pearson. Se calculó por cada correlación la regresión lineal de los datos.

\section{Grosor muscular}

Para el cálculo del grosor del MOE se usó la medición radiológica a nivel de los cortes de la tomografía axial computarizada (TAC) (fig. 1). Las mediciones empleadas fueron las correspondientes a las secciones musculares objetivadas a nivel del diámetro mayor del orificio herniario. Cada hemiabdomen se midió de manera 
independiente. Por cada paciente correspondían dos individuos a estudio.

\section{Medición de la tensión de la pared abdominal}

Aplicando la experiencia de otros autores ${ }^{10,-12}$, la medición de la tensión de la pared abdominal se realizó por medio de un dinamómetro de 100 newton de aluminio (referencia: 80098 MacroLinnie), con componentes internos de cloruro de polivinilo (PVC) y elementos de suspensión de acero inoxidable, reutilizable y esterilizado en el Hospital Comarcal de Zumárraga.

Durante la operación se fijó el borde fascial herniario por medio de una pinza de Cryle antes de la apertura de la vaina del recto abdominal y se procedió a la medialización del paquete muscular. La resistencia ejercida pudo medirse al enganchar el dinamómetro a los anillos de la pinza.

\section{RESULTADOS}

Los resultados demográficos de los individuos a estudio se expresan en la tabla 1.

Tres de los pacientes fueron intervenidos por hernia incisional, 2 de ellos con defectos paraumbilicales menores a $4 \mathrm{~cm}$ (M3W1, según la clasificación de la EHS) y un tercero con un defecto epigástrico ligeramente superior (M2W2, según la clasificación de la EHS). El cuarto paciente fue intervenido por una hernia umbilical primaria de mediano tamaño. En el $75 \%$ de los casos los pacientes mostraron una diástasis de rectos asociada (media de $4.83 \mathrm{~cm}, \pm 1.47$ ).

En el $75 \%$ de las intervenciones se realizó una eventroplastia retromuscular de Rives ${ }^{14}$ y en el $25 \%$, una reparación preperitoneal según la técnica de Bezama ${ }^{15}$.

La medición de la tensión de la pared abdominal y su correlación con el grosor del MOE arrojaron una correlación positiva entre el grosor del músculo y los newton medidos. El coeficiente de correlación de Pearson ( $r$ ) fue igual a 0.767, que demostró una 
correlación positiva, con un intervalo de confianza (IC) del $95 \%$ (0.136-0.955) y $\mathrm{p}$ de 0.026 (se muestra gráficamente en la figura 2 ). No obstante, únicamente el $58.9 \%$ de la variabilidad existente se explica por medio de dicha regresión.

En el caso de la correlación entre el grosor del paquete muscular completo y la fuerza medida de la pared, la correlación hallada fue positiva, con una $r$ de 0.272 (fig. 3), sin significación estadística. La correlación entre el grosor del MOI y el músculo transverso, por contra, obtuvo una correlación negativa de (-) 0.0604, sin obtener significación estadística en este caso tampoco. Los datos se exponen en la tabla II.

\section{DISCUSIÓN}

El futuro de la cirugía de la pared abdominal nace de la elección individualizada dentro del abanico de técnicas disponibles, sin olvidar que la pared abdominal es un juego de fuerzas mecánicas musculares que hay que intentar restaurar y reforzar, siempre que sea necesario, con mallas.

Siendo el MOE protagonista en la contención visceral del abdomen ${ }^{8,9}$, y conociendo la utilidad de su relajación para la cirugía de grandes defectos de pared abdominal ${ }^{3-6}$, el entendimiento individualizado de su fuerza podría aportar una información crucial en la reparación de hernias y de eventraciones.

Además, el proceso de cicatrización tisular habitual condena al tejido conectivo a semanas de regeneración. Se requieren meses e incluso años para su completa maduración ${ }^{13}$. De dicho proceso depende a su vez la integración tisular de las mallas protésicas, por lo que una modulación de las fuerzas mecánicas de la pared abdominal que disminuyese la tensión local podría facilitar una correcta consolidación de los tejidos y de la malla colocada.

Diversos estudios han tratado de medir la fuerza muscular durante el acto operatorio $0^{10-12}$ y han podido llegar a establecer una correlación entre la fuerza muscular abdominal y los newton 
hallados por medio de un dinamómetro ${ }^{12}$. No obstante, la medición requiere una instrumentación inexistente dentro del instrumental quirúrgico habitual; además, obliga a la utilización de la información obtenida durante la misma operación ${ }^{10}$. Poder conocer la tensión abdominal de manera preoperatoria adquiere una importancia estratégica dentro de la terapéutica de la hernia ventral e incisional.

Por medio de este estudio se ha establecido una correlación entre la fuerza muscular y su imagen preoperatoria. Se han obtenido, con una escasa $n$ muestral, resultados que arrojan una correlación significativamente positiva entre el grosor del MOE y la fuerza de la pared abdominal, sin que esta correlación haya podido observarse en el caso del resto de los músculos laterales del abdomen. No obstante, los resultados han de analizarse con cautela, ya que la bondad del estudio arroja un coeficiente de determinación inferior al 75-85\%. Se requieren más estudios para poder observar si la mayoría de los resultados puede explicarse por la regresión lineal obtenida.

Se deduce por tanto que, dentro de los músculos laterales del abdomen, el MOE es el que más fuerza añade a la línea media y que su tratamiento preoperatorio podría facilitar las reparaciones de la pared abdominal. Cabría esperar futuros estudios en los que se determinara a partir de qué grosor del MOE resultaría beneficioso aplicar tratamientos locales preoperatorios.

Por medio de este trabajo se vislumbran futuros en los que la aplicación de la toxina botulínica y la técnica quirúrgica elegida fueran individualizadas y seleccionadas para cada paciente en relación al grosor de su pared muscular, o más específicamente, del MOE.

\section{APROBACIÓN ÉTICA. CONSENTIMIENTO INFORMADO}

El procedimiento y el estudio se realizaron bajo el consentimiento firmado de todos los pacientes. Se les explicó la razón del estudio, posibles miras futuras y la inocuidad del procedimiento sin que se 
cambiara en ningún momento la intervención quirúrgica predispuesta.

Todos los procedimientos en este estudio se realizaron de acuerdo con las normas éticas del comité de investigación institucional o nacional y con la declaración de Helsinki de 1964 y sus enmiendas posteriores o normas éticas comparables.

\section{BIBLIOGRAFÍA}

1. Hernández-Granados $P$, López-Cano $M$, Morales-Conde $S$, Muysoms F, García-Alamino J, Pereira-Rodríguez JA. Profilaxis de la hernia incisional y utilización de mallas. Revisión narrativa. Cir Esp. 2018;96(2):76-87. DOI: 10.1016/j.ciresp.2018.01.003

2. Poulose BK, Shelton J, Phillips S, Moore D, Nealon W, Penson D, et al. Epidemiology and cost of ventral hernia repair: making the case for hernia research. Hernia 2012;16:179-83. DOI: 10.1007/s10029-011-0879-9

3. Ibarra-Hurtado TR, Nuño-Guzmán CM, Echeagaray-Herrera JE, Robles-Vélez E, de Jesús González-Jaime J. Use of botulinum toxin type a before abdominal wall hernia reconstruction. World J Surg. 2009;33(12):2553-6. DOI: 10.1007/s00268-0090203-3

4. Elstner KE, Read JW, Saunders J, Cosman PH, RodriguezAcevedo O, Jacombs ASW, et al. Selective muscle botulinum toxin A component paralysis in complex ventral hernia repair. Hernia. 2020;24(2):287-93. DOI: 10.1007/s10029-019-01939-3

5. Motz BM, Schlosser KA, Heniford BT. Chemical Components Separation: Concepts, Evidence, and Outcomes. Plast Reconstr Surg. 2018;142(Suppl3):58S-63S.

DOI: 10.1097/PRS.0000000000004856

6. Ibarra-Hurtado TR, Nuño-Guzmán CM, Miranda-Díaz AG, TroyoSanromán R, Navarro-lbarra R, Bravo-Cuéllar L. Effect of botulinum toxin type $A$ in lateral abdominal wall muscles 
thickness and length of patients with midline incisional hernia secondary to open abdomen management. Hernia. 2014;18(5):647-52. DOI: 10.1007/s10029-014-1280-2

7. Mesquita Montes A, Baptista J, Crasto C, de Melo CA, Santos R, Vilas-Boas JP. Abdominal muscle activity during breathing with and without inspiratory and expiratory loads in healthy subjects. J Electromyogr Kinesiol. 2016;30:143-50. DOI: 10.1016/j.jelekin.2016.07.002

8. Villalobos Mori RN. Estudio biomecánico del cierre de la pared abdominal. Lleida: Universitat de Lleida; 2020.

9. César Hijano J. Anatomía funcional de los músculos de la pared antero lateral del abdomen. Aspecto médico y quirúrgico. Buenos Aires: Universidad Nacional de La Plata; 2017.

10. Dragu A, Klein P, Unglaub F, Polykandriotis E, Kneser U, Hohenberger $W$, et al. Tensiometry as a decision tool for abdominal wall reconstruction with component separation. World J Surg. 2009;33(6):1174-80. DOI: 10.1007/s00268-0099991-8

11. Hope WW, Williams ZF, Rawles JW 3rd, Hooks WB 3rd, Clancy TV, Eckhauser FE. Rationale and Technique for Measuring Abdominal Wall Tension in Hernia Repair. Am Surg. 2018;84(9):1446-9.

12. Tenzel Paul L, Bilezikian Jordan A, Hope WW. Tension measurements in abdominal wall hernia repair: Concept and clinical applications. International Journal of Abdominal Wall and Hernia Surgery. 2019;2(4):119-24. DOI: 10.4103/ijawhs.ijawhs_37_19

13. Leong $M$, Murphy KD, Phillips LG. Capítulo 6: Cicatrización de las heridas Sabiston. Tratado de cirugía, Edición 20. Elsevier. pp. 130-62.

14. Rives J, Pire JC, Flament JB, Convers G. Traitement des éventrations. Encycl Med Chir. Paris. Techniques Chirurgicales. 1977. 4.2.07, 40165 . 
15. Bezama J, Debandi A, Haddad MM, Pablo U. Diástasis de rectos. Técnica quirúrgica original. Rev Chilena de Cirugía. 2009;61(1):97-100. DOI: 10.4067/S0718-40262009000100017

Tabla I. Datos demográficos de la muestra.

\begin{tabular}{|c|c|}
\hline Edad & $61.75( \pm 9.5)$ \\
\hline Sexo & 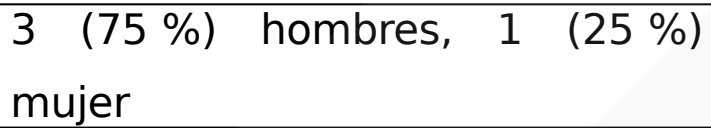 \\
\hline IMC & $31.9175( \pm 4.34)$ \\
\hline ASA I & $1(25 \%)$ \\
\hline ASA II & $1(25 \%)$ \\
\hline ASA III & $1(25 \%)$ \\
\hline ASA IV & $0(0 \%)$ \\
\hline DM & $0(0 \%)$ \\
\hline EPOC & $1(25 \%)$ \\
\hline Inmonosupresión & $0(0 \%)$ \\
\hline Malnutrición proteica & $0(0 \%)$ \\
\hline
\end{tabular}

Anesthesiologists Physical Status Classification System; DM: diabetes mellitus; EPOC: enfermedad pulmonar obstructiva crónica.

Tabla II. Correlación por cada grosor muscular.

\begin{tabular}{|l|l|l|l|}
\hline & \multicolumn{1}{|c|}{$\begin{array}{c}\text { Paquete } \\
\text { muscular } \\
\text { lateral }\end{array}$} & \multicolumn{1}{c|}{ MOE +} \\
transverso \\
\hline $\begin{array}{l}\text { Coeficiente de correlación, } \\
r\end{array}$ & 0.275 & 0.767 & $(-) 0.0604$ \\
\hline Intervalo al 95\% & $(-) 0.533-0.821$ & $0.136-0.955$ & $(-) 0.734-0.673$ \\
\hline Significación estadística & 0.509 & 0.026 & 0.887 \\
\hline $\begin{array}{l}\text { Coeficiente de } \\
\text { determinación, } \mathrm{R}^{2}\end{array}$ & $7.5 \%$ & $58.9 \%$ & $0.36 \%$ \\
\hline
\end{tabular}




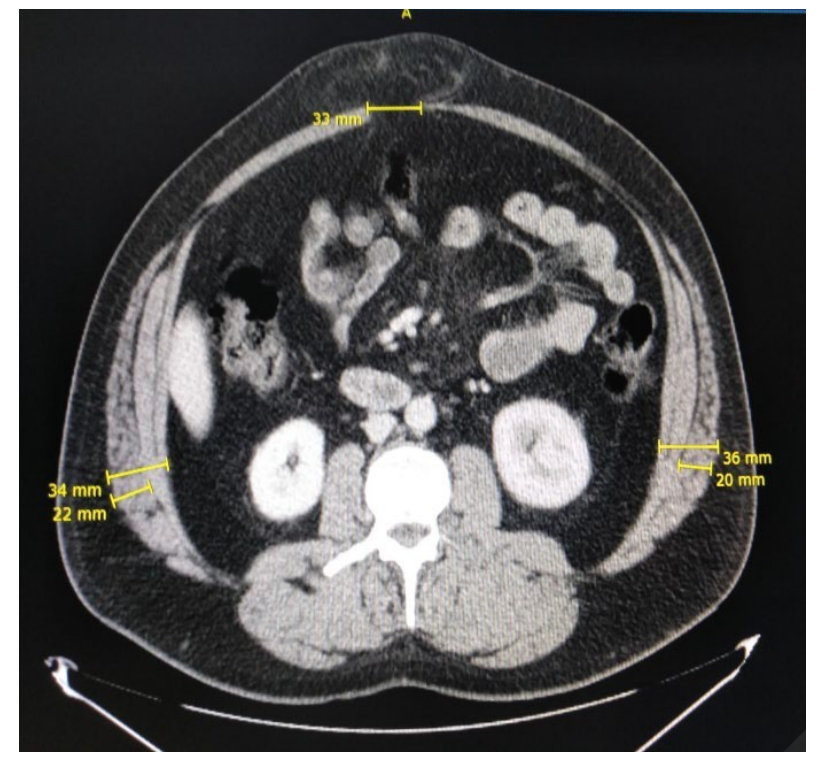

Figura 1. Ejemplos de medición en TAC.

CORRELACIÓN GROSOR/FUERZA

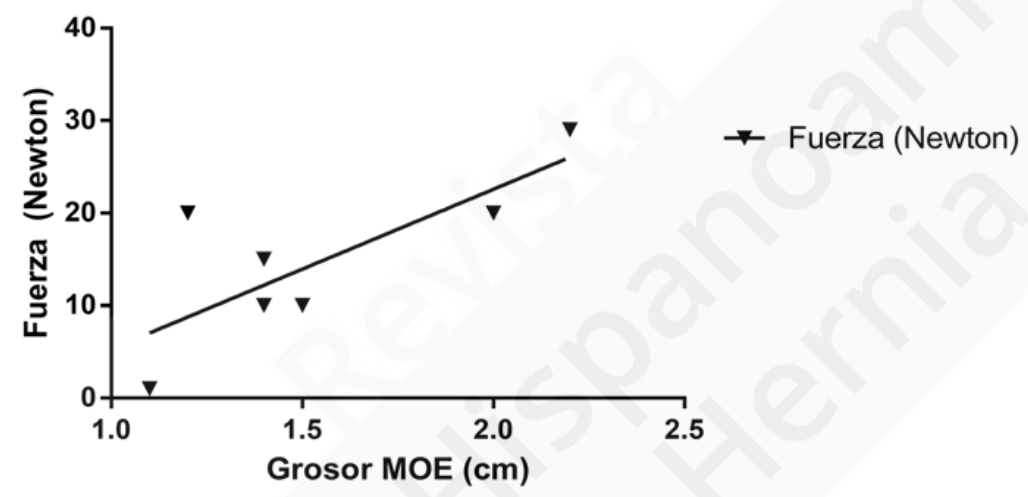

Figura 2. Representación gráfica de la correlación entre la fuerza medida en newton y el grosor del MOE. 
CORRELACIÓN GROSOR/FUERZA

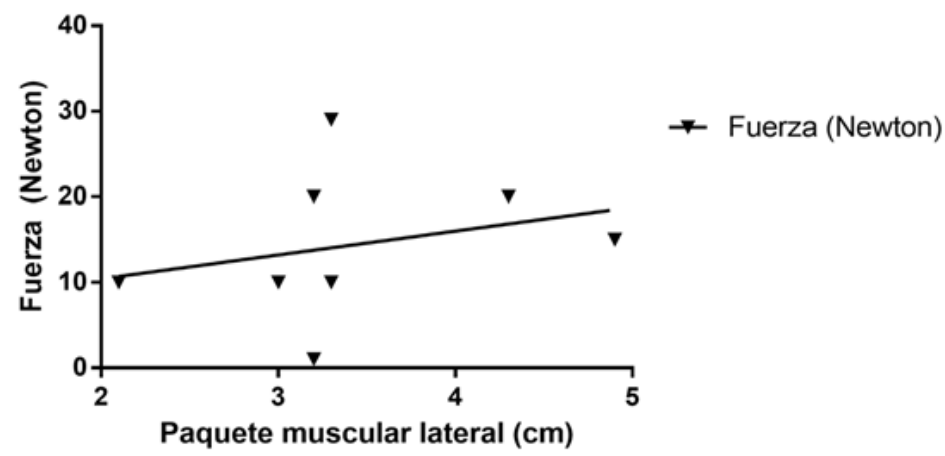

A

CORRELACIÓN GROSOR/FUERZA

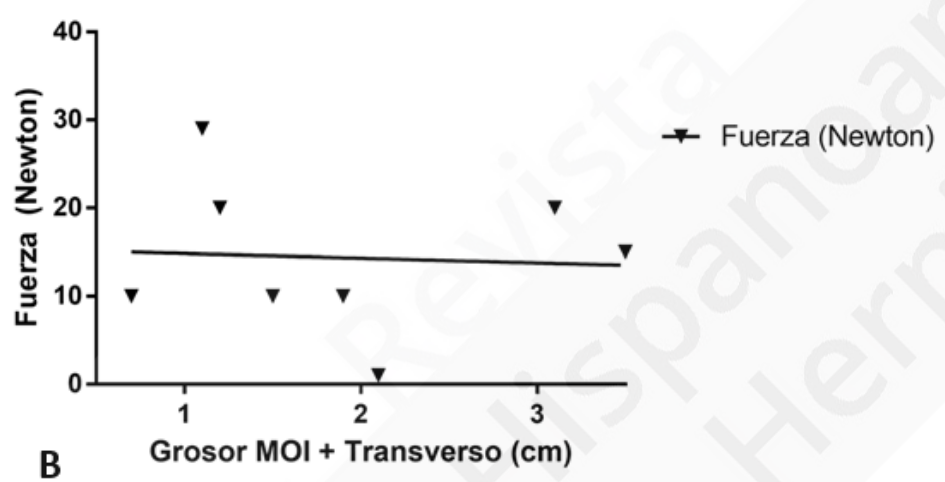

Figura 3. Representación gráfica de la correlación entre la fuerza medida y el grosor de todo el paquete muscular lateral (A) y del grosor del MOI y del músculo transverso (B). 